\title{
Possibilités de bouturage chez Maerua crassifolia Forssk., Capparaceae, un ligneux fourrager Sahélien
}

\author{
Sékouna DIATTA', Vilawoé Kayi HOUMEY', Leonard Elie AKPO'", \\ Chantal Yvette KABORE-ZOUNGRANA ${ }^{2}$, Maxime BANOIN ${ }^{3}$ \\ 'Laboratoire d'écologie végétale (UCAD/FST) BP 5005 Dakar (Sénégal) \\ ${ }^{2}$ Institut de développement rural(IDR/UPB), Bobo-Dioulasso (Burkina Faso) \\ ${ }^{3}$ Département Productions animales (UAM/FA) BP 10960 Niamey (Niger)
}

(Reçu le 07 Aout 2006, accepté le 12 Mars 2007)

*Correspondance,courriel: akpo@ird.sn

\section{Résumé}

Les caractéristiques du bouturage chez Maerua crassifolia, Forssk., Capparaceae un ligneux fourrager sahélien, et leurs variations mensuelles ont été établies avec des boutures issues de plantations obtenues par régénération artificielle. Sur un rameau de tige, nous avons distingué des boutures proximales, médianes et distales. Les travaux ont eu lieu durant la période sèche chaude, au cours des mois d'avril, mai, juin et juillet. Le délai de reprise est le même pour les différents types de boutures en avril. Pour ce mois, le taux de reprise le plus élevé $(86,70 \%)$ a été obtenu avec des boutures proximales avec une durée de reprise de 19 jours. Le taux de reprise le plus faible a été relevé au mois de mai, malgré une durée de reprise de 9 jours. Le mois de juillet, avec $91,30 \%$ de reprise et une durée de 19 jours parât le plus propice. De même le délai de reprise a été de 2 jours.

Mots-clés : Ligneux fourrager, bouturage, reprise, période, Sahel

\section{Abstract}

Possibilities of propagation by cutting at maerua crassifolia forssk., capparaceae, a sahelian fodder tree

Characteristics of the cutting of Maerva crassifolia, a sahelian fodder tree, and their monthly variations were established with cuttings resulting from plantations obtained by artificial regeneration. On a branch of stem, we distinguished closed, median and distant parts. Work took place during hot dry period, in May, April, June and July. The time of resumption is the same one for the various types of cuttings in April. For this month, the 
most elevated resumption rate $(86.70 \%)$ has been gotten with closed cuttings with a length of 19 days of resumption. The weakest resumption rate has been raised to the month of May, in spite of a length of 9 days of resumption. The month of July, with $91.30 \%$ of resumption and a length of 19 days appears most auspicious. In the same way the delay of resumption was of 2 days.

\section{Keywords : Fodder tree, cutting, resumption, period, Sahel}

\section{Introduction}

Ligneux très sobre et plastique [1], Maerua crassifolia est l'une des espèces adaptées à la fois aux sols pauvres et aux conditions de sécheresse. Elle est fréquente depuis les zones arides où les pluviométries annuelles sont inférieures à $100 \mathrm{~mm}$ [2] jusque dans les régions à pluviométrie annuelle de l'ordre de 300,400 voire $700 \mathrm{~mm}$. La plasticité de l'espèce lui permet ainsi de se développer dans diverses zones agroclimatiques.

Rencontrée de part et d'autre du Sahara et dans la péninsule arabique, en Afrique de l'Ouest et du Centre [3,4], M. crassifolia colonise des plaines, des dépressions et des dunes sableuses, des sols limoneux ou argileux.

En Afrique de l'Ouest, l'espèce est rencontrée principalement au Sahel, en zone sylvopastorale. Au Ferlo (Sénégal), l'arbuste, à l'état rabougri, ou sous forme de souche, est devenu rare. Au Niger, elle a été retrouvée dans l'Aïr (au Nord), très abondante dans la vallée de Dallol Bosso (à l'Est) et particulièrement à la station sahélienne expérimentale de Toukounouss dans le département de Filingué, où elle est parfois en peuplement quasi monospécifique ou en association avec Balanites aegyptiaca [5].

Les fevilles et les graines sont très appétées par le bétail. Les feuilles entrent dans l'alimentation des populations comme ingrédients culinaires; cela exacerbe la pression sur les arbustes.

A cela s'ajoute l'attaque des graines par des insectes avant leur maturité qui réduit considérablement les possibilités de régénération naturelle par voie sexuée [6].

Au Sahel, l'élevage est l'activité principale des populations. C'est un élevage traditionnel et de type extensif, dans lequel l'alimentation des animaux domestiques a toujours reposé sur les pâturages naturels. Abondant et de très bonne qualité pendant la saison des pluies, le pâturage herbacé, constitué exclusivement de plantes annuelles, est à l'état de paille en saison sèche, ou devient rare après le passage des feux. Cela constitue une contrainte majeure au développement de l'activité. 
Une bonne connaissance de l'espèce devra permettre de l'introduire dans les technologies agroforestières (banque fourragère, brise vent) afin d'accroître les effectifs de la population et de produire davantage de fourrage aérien.

\section{Matériel et méthodes}

\section{2-1. Présentation de Maerua crassifolia}

M. crassifolia (Photo I) est un arbuste sempervirent [7], dépassant rarement plus de 6 $\mathrm{m}$ de haut, avec un diamètre du tronc de l'ordre de $25 \mathrm{~cm}$. Le tronc porte des branches sarmenteuses et retombantes [2]. L'écorce est lisse, gris foncé, écailleuse chez les vieux sujets.

Les rameaux sont gris blanchâtres, le plus grand porte des nodosités sur lesquelles sont insérées les feuilles, alternes ou fasciculées, coriaces, glauques ou gris vert, obovales ou elliptiques, larges de 4 à $10 \mathrm{~mm}$ et longues de 9 à $20 \mathrm{~mm}$. Le pétiole est court (1 à $3 \mathrm{~mm}$ de long) elliptique voire obovale acuminé, pouvant être même légèrement échancré [8]; Il est prolongé par une feville à limbe ovale de 10 à $20 \mathrm{~mm}$ de long sur 5 à $10 \mathrm{~mm}$ de large. Les nervures pennées sont peu saillantes.

La floraison a lieu entre février et Mars, correspondant à la fin de la saison sèche froide et début de la saison sèche chaude. Les fleurs solitaires ou disposées par 2 ou 3 dans les fascicules des feuilles sont blanches à verdâtres, parfois jaunes, à pédicelles et calices pubescentes atteignant $2 \mathrm{~cm}$ de long [9]. Les fleurs dégagent un parfum suave.

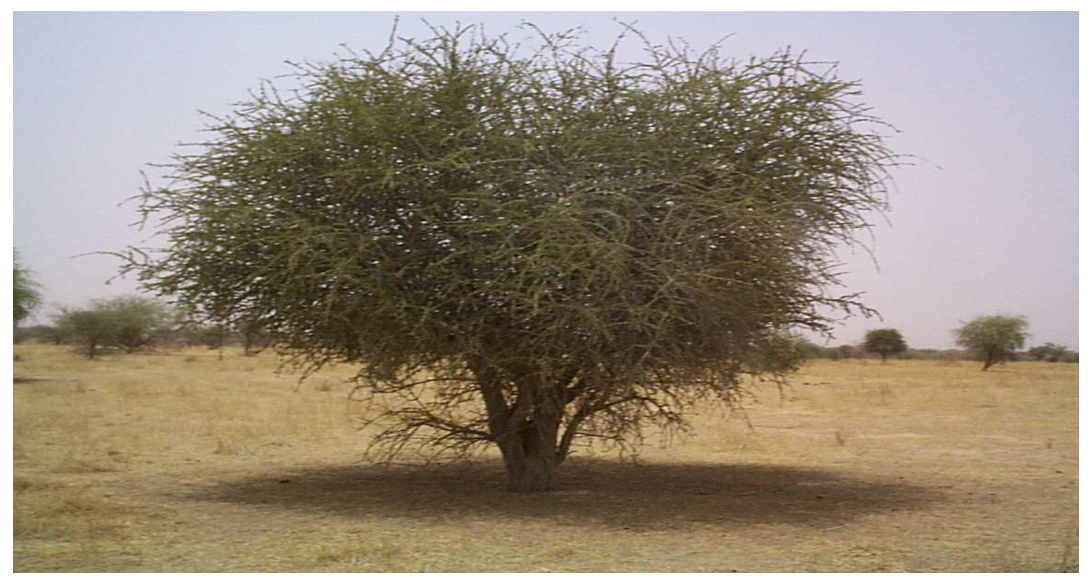

Photo 1 : Arbuste de Maerua crassifolia Forssk., (Capparaceae) à la Station sahélienne expérimentale de Toukounous (Niger). Cliché : Diatta, février 2004. 
Les fruits sont des gousses brunes allongées, fortement étranglées entre les graines longues de 3 à $6 \mathrm{~cm}$, finement pubescentes et apparaissent en avril [10].

Les feuilles, riches en $\beta$-carotène [11] et les fruits, qui constituent un excellent fourrage durant la saison sèche [12], sont particulièrement des sources de protéines et d'éléments minéraux [13].

Les rameaux verts sont broutés par tous les animaux domestiques et sauvages. II semble toutefois que les chevaux et les ânes n'en consomment pas ou très peu. Le chameau aime les fleurs. C'est une plante rustique, résistante au broutage [14].

Les fruits sont combustibles. Les feuilles très riches en calories sont utilisées en pharmacopées pour les coliques. Elles sont aussi mangées avec le couscous.

L'écorce est utilisée contre la fièvre, la carie dentaire, ou pour purifier l'eau. Réduite en cendre, l'écorce est utilisée pour faire de la teinture noire [9].

Maerua crassifolia apparaît ainsi comme un arbre à usages multiples, une espèce agroforestière.

\section{2-2. Origine des boutures}

Les semences ont été prélevées à partir des plantations de $M$. crassifolia installées de 1992 et 1998 dans la parcelle expérimentale de la Faculté d'Agronomie de l'Université Abdou Moumouni (UAM) de Niamey (Niger), située à $13^{\circ} \mathrm{N}$ et à $2^{\circ} 08^{\prime} \mathrm{E}$ [15].

Sur un fragment de branche, nous avons distingué 3 parties ou types de bouture selon leur position sur le rameau prélevé (Figure I), il s'agit des parties (ou boutures) distale (bd), médiane (bm) et proximale (bp).

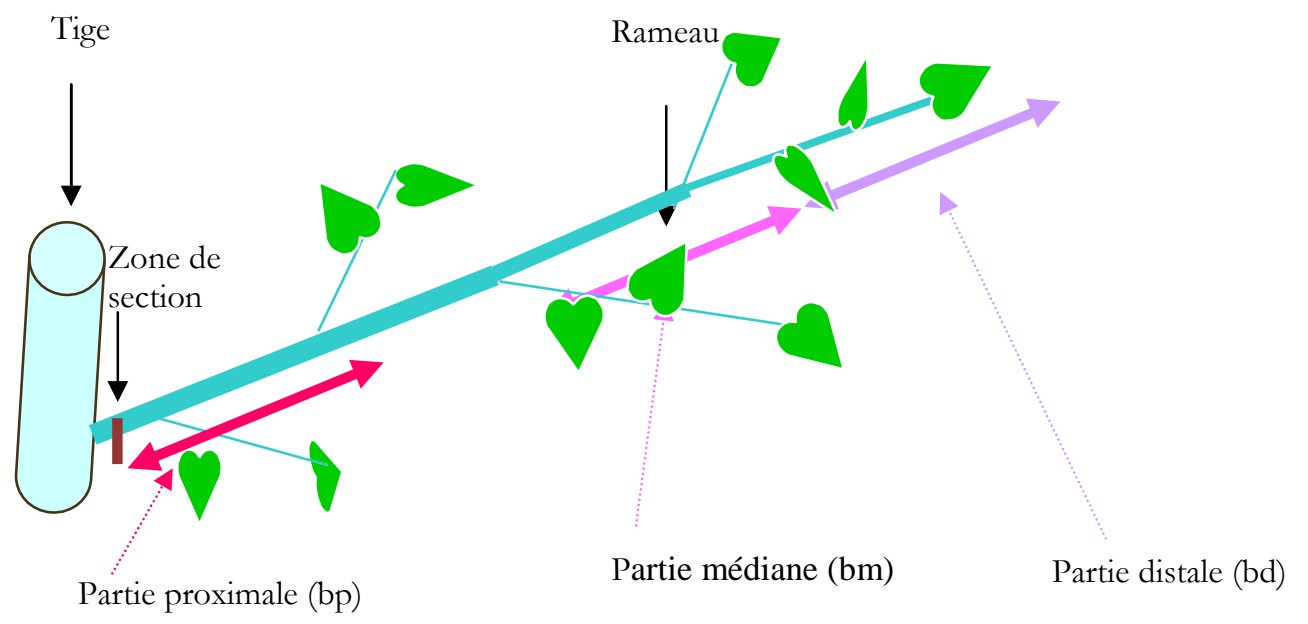

Figure 1 : Position des différents types de bouture sur un rameau de M. crassifolia 


\section{2-3. Culture des boutures}

Les boutures ont été plantées dans des pots de culture constitués de sachets plastiques en polyéthylène et installés dans la parcelle. Pour chaque type de bouture, nous avons utilisé 20 fragments de tige longs de $15 \mathrm{~cm}$ chacun avec 3 répétitions, soit 60 boutures par type et 180 boutures pour les trois parties précédemment définies (bp, bm, bd).

La période sèche et chaude permet une bonne reprise des boutures (Danthu : com. verbale). Ainsi nous avons planté les boutures au cours des mois d'avril, mai, juin et juillet.

Le suivi des boutures a lieu tous les jours pendant un mois. La reprise d'une bouture est caractérisée par l'apparition d'un bourgeon caulinaire.

\section{2-4. Traitement des données et expression des résultats}

Nous avons défini les caractéristiques suivantes:

- le délai de reprise : temps qui s'écoule entre la mise en terre de la bouture et l'apparition du premier bourgeon;

- la durée de reprise : temps qui s'écoule entre l'apparition du premier bourgeon et celle du dernier bourgeon;

- le taux de reprise des boutures: rapport entre le nombre de boutures ayant donné de bourgeons et le nombre total de boutures plantées; le taux maximal de reprise correspond à la capacité de reprise ou capacité de bouturage de l'espèce;

- la vitesse de reprise : variation du taux de reprise des boutures en fonction du temps. Rapport entre le nombre cumulé de boutures ayant donné de bourgeons quotidiennement et le nombre de boutures plantées.

Pour ces différents paramètres, nous avons soit considéré des périodes, soit calculé des moyennes associées au coefficient de variation, soit enfin comparé des moyennes par des tests de $X^{2}$ ou d'Anova [16].

\section{Résultats}

\section{3-1. Caractéristiques du bouturage}

Le Tableau $I$ présente le temps de latence et la durée de reprise des boutures (Photo 2). 
Tableau 1 : Variation du temps de latence et de la durée de reprise des boutures de M. crassifolia

\begin{tabular}{|c|c|c|c|}
\hline Fragments de $15 \mathrm{~cm}$ & Proximal & Médian & Distal \\
\hline Temps de latence (jours) & 4 & 4 & 4 \\
\hline Durée de reprise (jours) & 19 & 22 & 23 \\
\hline
\end{tabular}

\section{3-1-1. Le délai de reprise ou temps de latence}

Le temps de latence est le même pour les différentes parties des fragments de tige ou de rameau, il est de 4 jours. C'est en effet à partir du $5^{\text {ème }}$ jour que nous avons enregistré l'apparition des bourgeons au niveau des boutures : 2 dans le groupe des boutures proximales, 1 dans chacun des autres parties (médiane et distale).

\section{3-1-2. La durée de reprise}

La durée de reprise a varié de 19 à 23 jours, soit en moyenne 22 jours. Elle est relativement plus courte dans les parties proximales et plus longue vers les parties distales.

\section{3-1-3. La capacité de bouturage ou le taux de reprise}

Le Tableau 2 présente la variation de la capacité de bouturage de $M$. crassifolia en fonction des lots et de types de bouture.

Dans tous les lots, nous n'avons pas observé une reprise totale des boutures. Les valeurs de reprise maximale ont été observées dans les parties proximales (95\%).

Tableau 2 : Variation de la capacité de bouturage (\%) de M. crassifolia en fonction des lots et des types de bouture

\begin{tabular}{|l|c|c|c|}
\hline \multicolumn{1}{|c|}{ Fragments } & Proximal & Médian & Distal \\
\hline Lot 1 & 95 & 80 & 80 \\
\hline Lot 2 & 75 & 60 & 55 \\
\hline Lot 3 & 90 & 80 & 65 \\
\hline Moyenne (\%) & 86,7 & 73,3 & 66,7 \\
\hline Coefficient de variation (Cv \%) & 12,01 & 15,75 & 18,9 \\
\hline X $^{2}$ & \multicolumn{3}{|c|}{23,54} \\
\hline d.d.l. & \multicolumn{3}{|c|}{} \\
\hline
\end{tabular}


Lorsque nous considérons toutes les parties ensemble, la capacité de bouturage de $M$. crassifolia est de l'ordre de 75,6\%. La capacité de bouturage de $M$. crassifolia a présenté des variations selon le type de bouture :

- de 75 à $95 \%$ pour les boutures proximales;

- de 60 à $80 \%$ dans le groupe des boutures médianes;

- de 55 à $80 \%$ pour les boutures distales,

soit en moyenne $86,7 \% ; 73,3 \%$ et $66,7 \%$ respectivement.

Le test de $X^{2}$ appliqué à ces différents taux de reprise a donné une valeur de test $(23,547$ ; $\mathrm{ddl}=4$ ) largement supérieure à celle de $\alpha_{0,01}(13,277)$ voire de $\alpha_{0,001}(18,467)$ révélant ainsi une différence hautement significative.

Nous avons pu ainsi distinguer en fonction le long du rameau prélevé :

- les parties proximales

- les parties médianes

- les parties distales,

c'est-à-dire des parties proches du tronc vers l'extrémité. La capacité de bouturage de l'espèce diminue au fur et à mesure que l'on s'éloigne de la tige.

La capacité de bouturage apparâ̂t aussi inversement proportionnelle à la durée de reprise. Ainsi plus la durée de reprise est brève, plus élevée est la capacité de bouturage.

\section{3-1-4. La vitesse de reprise}

La vitesse de reprise, qui traduit la variation journalière de la reprise est représentée par la Figure 2.

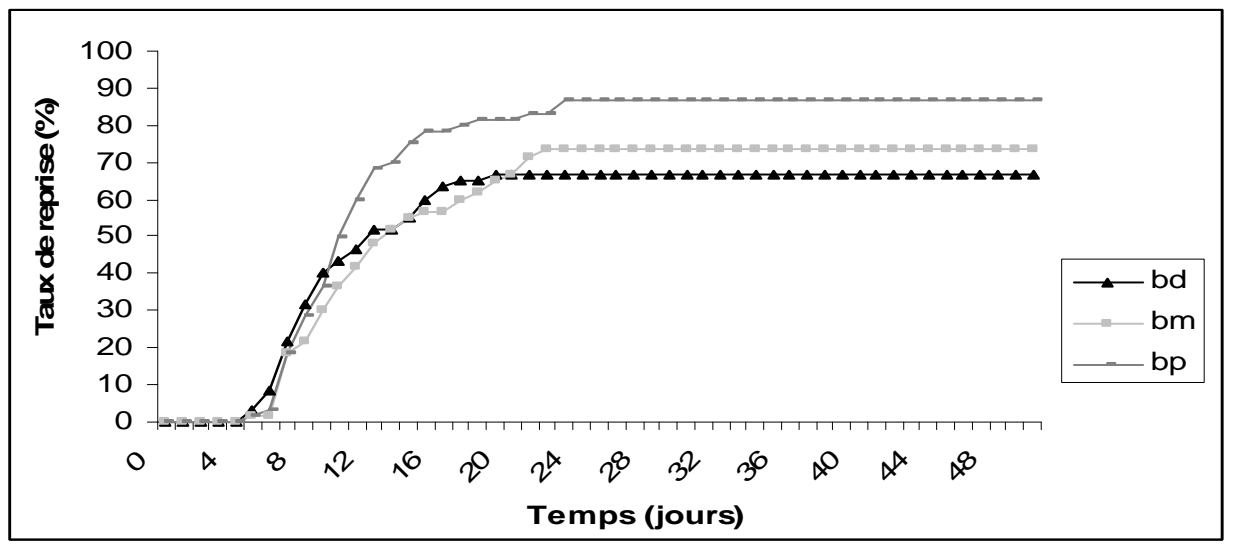

Figure 2 : Évolution du taux de reprise des boutures distales (bd), médianes (bm) et proximales (bp) de M. crassifolia en fonction du temps. 
L'allure des courbes présente les mêmes tendances quel que soit le type de bouture. Les différences sont apparues à partir du $10^{\text {òme }}$ jour : beaucoup plus de reprises dans le groupe des boutures proximales (24 soit $40 \%$ ), suivies des boutures distales ( $36 \%$ ) et médianes $(30 \%)$. Cette situation a été maintenue jusqu'à la $3^{3 \text { ème }}$ semaine. A partir de cette semaine, nous n'avons pratiquement plus enregistré de reprises.

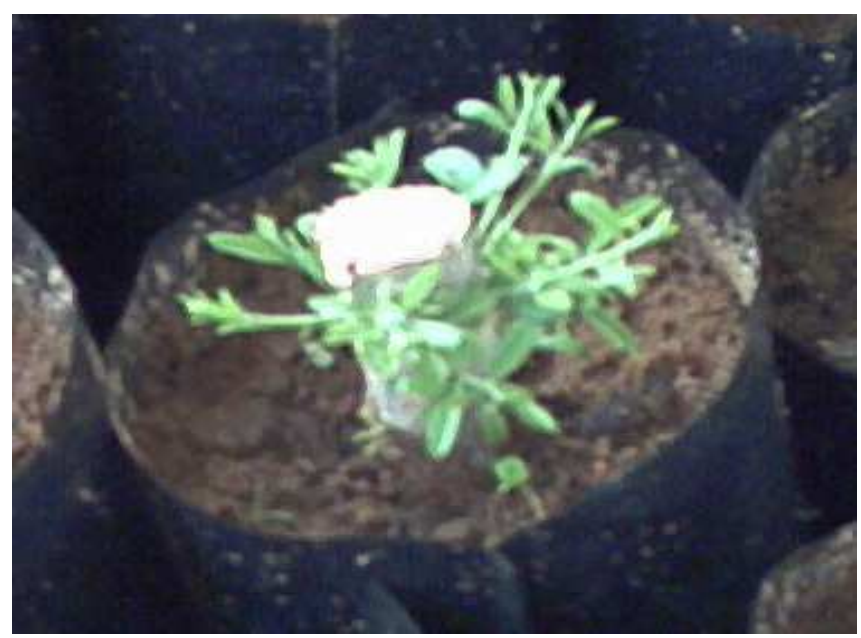

Photo 2 : Jeune plant de M. crassifolia âgé de 70 jours issu de bouture de tige

La proportion de boutures proximales ayant repris a été la plus élevée, 86,7 \% ; c'est ce fragment que nous allons utiliser dans la suite des expérimentations.

\section{2-2. A la recherche de la période optimale de reprise}

Nous avons analysé les caractéristiques des boutures de type basal de $M$. crassifolia (Tableau 3) au cours de la saison favorable (saison sèche et chaude).

Tableau 3 : Caractéristiques des boutures de type basal de M. crassifolia

\begin{tabular}{|l|c|c|c|}
\hline \multicolumn{1}{|c|}{ Mois } & Délai (jours) & Durée (jours) & Capacité (\%) \\
\hline Avril & 4 & 19 & 86,7 \\
\hline Mai & 3 & 9 & 24,7 \\
\hline Juin & 2 & 39 & 94,0 \\
\hline Juillet & 2 & 19 & 91,3 \\
\hline Moyenne & 3 & 22 & 74,2 \\
\hline
\end{tabular}




\section{2-2-1. La capacité de reprise}

Durant la période retenue, sèche et chaude, le délai moyen de reprise a été de 3 jours. La durée de reprise a varié de 9 jours dans le mois de mai à 39 jours en juin, soit en moyenne 22 jours.

Le taux moyen de reprise des boutures durant la période sèche et chaude est de $74,2 \%$. Il paraît relativement élevé. A l'exception du mois de mai $(24,7 \%)$, le taux de reprise a toujours été supérieur a celui du mois d'avril $(86,7 \%)$. Nous avons enregistré le taux élevé dans les essais du mois de juin (94\%) avec une durée de reprise relativement longue (39 jours). Dans le mois de juillet en revanche, nous avons observé $91,3 \%$ de reprise en 19 jours.

En avril et juillet, la durée de reprise est la même (19 jours) mais le taux de reprise est nettement meilleur en juillet $(+3,6 \%)$.

L'examen du tableau a fait ressortir aussi que la durée de reprise a une influence sur la capacité. En effet, plus la durée est longue, meilleure paraît la capacité de reprise.

\section{3-2-2. La vitesse de reprise}

La vitesse de reprise des boutures de type proximal pendant la période d'étude est présentée sur la Figure 3.

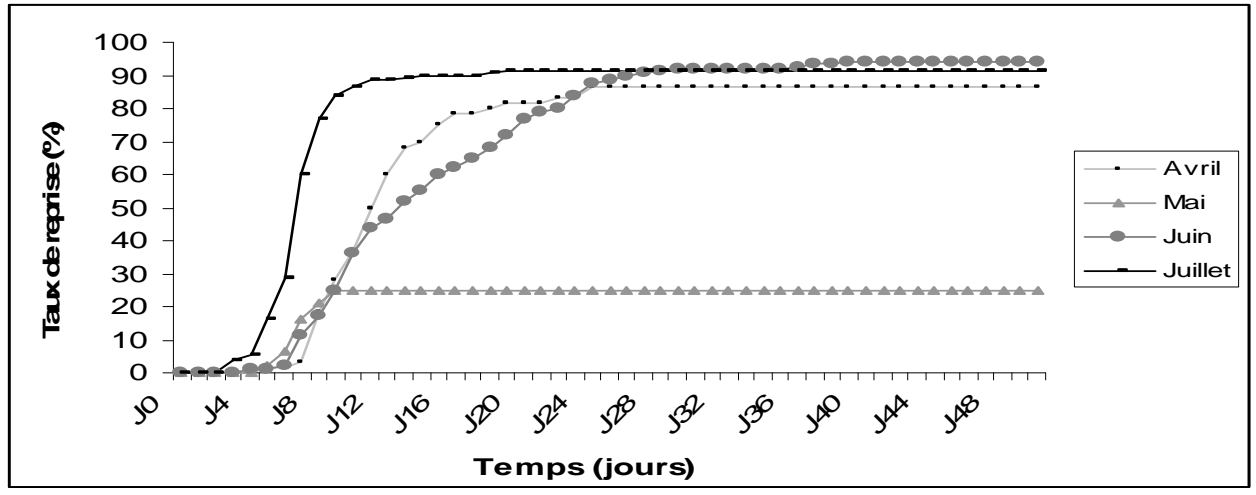

Figure 3 : Evolution journalière du taux de reprise des boutures proximales de M. crassifolia en culture entre les mois d'avril et de juillet

La reprise est caractérisée par l'apparition de bourgeons au niveau de 2 boutures au mois d'avril, 3 en mai, 2 en juin et 6 en juillet, soit 1,$67 ; 2 ; 1,33$ et $4 \%$ respectivement pour atteindre à la fin de la première semaine 3,$3 ; 16 ; 11,3$ et $60 \%$. Le maximum de 
boutures ayant repris est atteint en 15 jours de reprise. Cela traduit bien le caractère groupé du phénomène, qui est d'ailleurs exacerbé durant le mois de juillet.

Le mois de juillet parâ̂t ainsi le mois le plus propice pour le bouturage de l'espèce étudiée : $90 \%$ de reprise en 19 jours.

\section{Discussion}

Ce travail a étudié le bouturage chez $M$. crassifolia. Trois principales parties de rameau prélevé ont été distinguées ; il s'agit des parties proximale, médiane et distale. Chacune des parties a constitué une modalité ou type de bouture. Les différents types de boutures ont été plantés dans des pots de culture. Les observations ont porté sur le temps de latence, la durée de reprise des boutures et la capacité de bouturage de l'espèce.

Les principales caractéristiques de bouturage de l'espèce ont permis d'indiquer que :

- 1) le temps de latence est partout uniforme ; il ne dépend donc pas du type de bouture ;

- 2) la durée de reprise augmente de la base du rameau vers l'extrémité alors que la capacité de reprise évolue dans le sens contraire ; elle décrô̂t des parties proximales vers les parties distales;

- 3) la capacité de bouturage ne dépend que de la position du fragment sur le rameau ; elle est meilleure avec les fragments de type basal.

Ces observations, qui concordent avec celles rapportées par Salifou [17], présentent toutefois des valeurs nettement plus élevées.

S'agirait-il de la période utilisée par cet auteur ? Le diamètre des boutures utilisées ne pourrait-il aussi entraîner des différences?

La reprise d'une bouture est due à une relance d'activité au niveau d'un ou de plusieurs bourgeons végétatifs qui vont donner plus tard un rameau. Chaque bourgeon végétatif possède un point végétatif qui est relativement vulnérables aux chocs ou à la dessiccation, car il est composé de tissu de caractère méristématique, c'est-à-dire de cellules jeunes, non différenciées, capables de multiplication rapide [18]. Plusieurs facteurs pourraient être à l'origine du caractère décroissant du taux de reprise des boutures de la base vers le sommet des branches.

D'abord chez $M$. crassifolia, les rameaux sont caractérisés par la présence de nodosités sur lesquelles sont insérées les feuilles, alternes ou fasciculées. Or le nombre de ces nodosités qui sont le siège des bourgeons végétatifs décroit de la partie proximale à celle apicale, d'où le gradient décroissant du taux de reprise. Ensuite, les bourgeons végétatifs en faible nombre chez les boutures de types médian et apical pourraient être affectés par le dessèchement de l'épiderme, entraînant ainsi la mort de ces dernières. 
Enfin, physiologiquement, la dominance apicale (due à l'acide $\beta$-indolylacétique ou AIA) exercée par le bourgeon terminal sur les bourgeons axillaires étant levée pour chaque type de bouture, la balance hormonale (auxines-cytokinines) qui conditionne pour une grande part l'apparition de bourgeons serait optimale chez les boutures de type proximal.

Aussi la vitesse de reprise est accélérée à partir de la deuxième semaine. On pourrait penser que c'est le temps où la plupart des boutures se seraient remises du stress causé par leur isolement du pied mère.

Bien que faible, le délai de reprise des boutures proximales a vairé entre les mois d'observation ; les délais des mois de mai et juillet ont été les plus brefs ( 2 jours). Ces mois ont présenté aussi la durée la plus brève mais les capacités de reprise totalement différentes. Le mois de mai est mois de chaleur sèche alors que le mois de juillet est associé à des températures plus douces, une humidité relative plus élevée avec apparition de certaines espèces herbacées, témoignant des conditions de milieu plus favorables.

Aussi des facteurs endogènes liés surtout à l'état physiologique de la plante pourraient favoriser la reprise.

Signalons enfin qu'une succession de pluies au cours du mois de mai provoque le pourrissement des boutures en raison de la chaleur humide; cela pourrait expliquer le très faible taux de reprise observé au cours de ce mois. Le Sahel étant caractérisé entre autres par une pluviométrie aléatoire, l'eau constitue une contrainte majeure pour la production de jeunes plants en pépinières et leur survie en milieu réel. Ainsi la production et la survie des jeunes plants ne peuvent être envisagées que dans des zones où l'eau est accessible. C'est le cas par exemple des alentours des points ou cours d'eau dans les parcours naturels ou en zone périurbaine, siège d'un élevage en plein essor. Dans ce dernier cas, les eaux usées peuvent être utilisées en appoint après traitement pour l'arrosage des jeunes plants, soit par les méthodes classiques soit par le système d'irrigation au goutte-à-goutte. Pour promouvoir le développement de l'élevage en particulier et de l'agriculture en général, la maîtrise de l'eau semble être une condition sine qua non.

\section{Conclusion}

L'étude a permis de retenir les principales caractéristiques suivantes du bouturage de Maerva crassifolia : 
- Ie délai de reprise des boutures est généralement faible, encore plus faible au cours du mois de juillet; il n'a pas varié pas en fonction du type de boutures;

- la durée des reprises est d'environ 3 semaines;

- la capacité de reprise est plus élevée chez les boutures proximales, particulièrement au cours du mois de juin et juillet ;

- le mois de juillet paraît toutefois propice pour produire des plants de Maerua crassifolia issus de boutures avec un délai de reprise particulièrement bref.

\section{Remerciements}

Ce travail est le fruit de la coopération inter-universitaire (entre l'Université Abdou Moumouni de Niamey, I'Université Cheikh Anta Diop de Dakar et l'Université polytechnique de Bobo Dioulasso). II a bénéficié du soutien, combien précieux, de l'AUF.

\section{Références}

[1] - G. BOUDET, Manuel sur les pâturages tropicaux et les cultures fourragères.

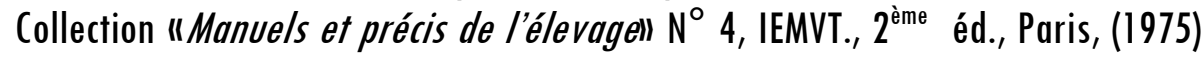
$254 \mathrm{p}$.

[2] - H. J. MAYDELL (von), Arbres et Arbustes du Sahel: Leurs caractéristiques et leurs utilisations. Eschbom, FR Germany, GTZ (1983) $530 \mathrm{p}$.

[3] - M. G. CURASSON, Etudes sur les pâturages tropicaux et subtropicaux. II. Les pâturages des principales régions. Rev. Elev. Méd. vét. Pays trop., 7, 2 (1954) 103-120.

[4] - F. WHITE, La végétation africaine : mémoire accompagnant la carte de la végétation de l'Afrique Unesco/AETFAT/UNSO (1986) $384 \mathrm{p}$.

[5] - S. DIATtA, S. DOUMA, M. ChaNONO, M. BANOIN, C. Y. KABORE-ZOUNGRANA, A. TOUDOU, L. E. AKPO, Caractéristiques de Maerua crassifolia Forssk., ligneux fourrager des terres de parcours sahéliennes (Toukounous - Filingué, Niger). Rev.afr. Santé et Productions animales 2, 2 (2004) 148-153.

[6] - S. DIATTA, I. SALIFOU, M. O. SY, C. Y. KABORE-ZOUNGRANA, M.BANOIN, L. E. AKPO, Évaluation des potentialités germinatives d'un ligneux fourrager sahélien : Maerua crassifolia Forssk., Capparaceae. (Rev. Sécheresse : Sous presse).

[7] - J. BERHAUT, Flore illustrée du Sénégal. Dicotylédones. Tome II. Balanophoracées à Composées. Gouvernement du Sénégal-Ministère du développement Rural et de I'Hydraulique. Direction des Eaux et Forêts. Dakar, (1974) 695 p. 
[8] R. BARTHA, Plantes fourragères de la zone sahélienne d'Afrique, Afrika Studien Nr. 48, Weltforum Verlag, München, (1970) $306 \mathrm{p}$.

[9] M. ARBONNIER, Arbres, arbustes et lianes des zones sèches d'Afrique de l'ovest, CIRAD, MNAM, UICN, (2000) $542 \mathrm{p}$.

[10] M. BAUMER, Arbres, arbustes et arbrisseaux en Afrique occidentale, Série Etudes et Recherches Enda-Editions Dakar, (1995) 168-170

[11] G. BERGE et B. HVEEM, Valeur nutritive de quelques plantes alimentaires sauvages dans le Gourma. Annexe $n^{\circ} 13$ du Rapport d'étape sur les Plantes sauvages du Projet de Recherche SSE, Environnement et Développement au Mali, (1992) 26 p.

[12] F. ACHARD, S. BOULKACIM et A. GOURO, La Station sahélienne d'élevage de Toukounous au Niger : Productivité d'un système de production extensif amélioré. In : Gaston A., Kernick M., Le Houérou H.N., ed., Actes IVe Congr. int. Terres de Parcours, vol. 2, Montpellier, France, 22-26 avril 1991. Montpellier, France, CIRAD, 775-778.

[13] H. N. LE HOUEROU, Le rôle des ligneux fourragers dans les zones sahélienne et soudanienne. In : Les fourrages ligneux en Afrique. Etat actuel des connaissances. Addis-Abeba, CIPEA, (1980) $481 \mathrm{p}$.

[14] - M. I. CISSE, Production fourragère de quelques arbres sahéliens : relation entre biomasse foliaire maximale et divers paramètres physiques, in "Les fourrages ligneux en Afrique. Etat des connaissances", CIPEA, Addis Abeba, (1980) 203-208

[15] - A. YENIKOYE, Etude de l'endocrinologie sexuelle et de la croissance folliculaire chez la brebis nigérienne de race Peulh : influence de la saison de reproduction. Doct. d'Etat ès Sc. nat. Univers. Tours, (1986) $91 \mathrm{p}$.

[16] - D. SCHWARTZ, Méthodes statistiques à l'usage des médecins et des biologistes, Flammarion Médecine-Sciences, $3^{\mathrm{e}}$ édition (1969) $318 \mathrm{p}$.

[17] I. SALIFOU, Contribution à la domestication de deux espèces fourragères spontanées Alysicarpus ovalifolius (Schum. et Thorn.) J. Léonard et Maerua crassifolia Forsk. Doctorat de $3^{\mathrm{e}}$ cycle. U A M, Niamey, (2002) 124 p.

[18] P. ROLIN, Bourgeons. In Encyclopédia Universalis et A. Michel: Dictionnaire de la Botanique, (1999) $1511 \mathrm{p}$. 